most abundant leukocytes in Human blood, but it is not clear whether neutrophils exert an important role in the pathogenesis of organ tissue damage in SLE.

Methods We used lupus-prone mouse model and model of lupus serum-induced tissue inflammation in mouse to investigate the role of neutrophils in the organ damage of SLE.

Results We found that there was a little neutrophil infiltration in the inflammatory sites of skin, liver, brain and joint in lupus-prone mice. We also found that there was also little neutrophil infiltration in the site of skin inflammation induced by lupus serum in normal mouse. The severity of skin inflammation induced by lupus serum was not significantly decreased in mice with neutrophil depletion compared to ones without neutrophil depletion. But we found that neutrophils were actually involved in tissue injury induced by lupus IgG. Further studies showed that lupus IgG stimulated and activated neutrophils, and cause the death of neutrophils. Studies also confirmed that Fas plays an important role in neutrophils apoptosis.

Conclusions Our study indicates that neutrophils participate in the early stage of lupus organ damage, and then they died through activation-mediated apoptosis. These findings promote the understanding of the role of neutrophils in the tissue injury with SLE.

\section{TLR8 ACTIVATION IN NEUTROPHILS IMPAIRS IMMUNE COMPLEX PHAGOCYTOSIS THROUGH FURIN- DEPENDENT SHEDDING OF FCGRIIA}

K Elkon*, S Arve, J Ledbetter, C Lood. University of Washington, Medicine, Seattle, USA

\subsection{6/lupus-2017-000215.340}

Background and aims Neutrophils play a crucial role in host defense through mechanisms including phagocytosis and formation of neutrophil extracellular traps (NETs), a recently identified neutrophil cell death process in which DNA is extruded together with cytoplasmic and granular content to trap and eliminate extracellular pathogens. Although beneficial from a host-pathogen perspective, exaggerated neutrophil activation has been linked to autoimmunity, in particular the rheumatic disease systemic lupus erythematosus (SLE) where nucleic acid-containing immune complexes (IC) drive inflammation. Toll-like receptor (TLR) agonists, such as nucleic acids, are important components of pathogens, enabling enhanced phagocytosis by macrophages and dendritic cells, but the role of TLR signalling in processing of SLE ICs and downstream inflammatory neutrophil effector functions is not known.

Methods Standard Methods.

Results We observed that both FcgR- and TLR8-engagement were required for induction of NETosis, whereas TLR8 activation, through the RNA component of the ICs, suppressed further IC-mediated phagocytosis. Mechanistically, TLR8 ligation induced PI3K-dependent ROS generation through NADPH oxidase, and subsequent furin-dependent proteolytic cleavage of the N-terminal part of FcgRIIA shifting neutrophils away from phagocytosis of ICs toward NETosis. TLR8 activated neutrophils promoted cleavage of FcgRIIA also on plasmacytoid dendritic cells and monocytes resulting in impaired overall clearance of ICs and increased complement C5a generation. Importantly, ex vivo derived activated neutrophils from SLE patients demonstrated a similar cleavage of FcgRIIA that was correlated with markers of disease activity as well as complement activation.

Conclusions Therapeutic approaches aimed at blocking TLR8 activation would be predicted to increase phagocytosis of circulating ICs while disarming their inflammatory potential.

\section{CD11B REGULATES INFLAMMATION, AUTOIMMUNITY AND ASSOCIATED PATHOLOGY IN A MODEL OF SYSTEMIC LUPUS ERYTHEMATOSUS}

T Gottschalk*, E Tsantikos, M.L. Hibbs. Monash University, Department of Immunology and Pathology, Melbourne, Australia

\subsection{6/lupus-2017-000215.341}

Background and aims Systemic Lupus Erythematosus (SLE) is a highly complex, heterogeneous autoimmune disease characterised by circulating self-reactive antibodies that deposit in tissues including skin, kidneys and brain, alongside a chronic inflammatory response that leads to progressive tissue damage and impaired function. Genome-wide association studies have identified a number of receptors and signal transduction molecules specific for the immune system that predispose to the development of SLE. A loss-of-function single nucleotide polymorphism (SNP) in the Itgam gene encoding CD11b (rs1143679) has been identified which associates with an increased incidence of SLE, implicating CD11b as a protective factor against disease development. To understand the role that $\mathrm{CD} 11 \mathrm{~b}$ plays in controlling autoimmune disease, we crossed CD11b deficient mice $\left(\mathrm{CD} 11 \mathrm{~b}^{-/-}\right)$with Lyn deficient $\left(\mathrm{Lyn}^{-/}\right)$mice, a well-studied, robust model of human SLE.

Methods Double knockout $\mathrm{Lyn}^{-/-} \mathrm{CD} 11 \mathrm{~b}^{-/-}$mice were analysed over time for development of autoimmune disease and inflammation.

Results While CD $11 b^{-/-}$mice presented with mild splenomegaly and lymphadenopathy, immune cell compartments were unchanged and pathogenic IgG anti-dsDNA autoantibody titres and glomerulonephritis were undetected suggesting that $\mathrm{CD} 11 \mathrm{~b}$ deficiency alone is insufficient to drive autoimmune disease. Conversely, deficiency of CD11b on the Lyn-deficient autoimmune-prone background exacerbated disease, driving splenomegaly and lymphadenopathy, extramedullary haematopoiesis, autoantibody production and glomerulonephritis, which heavily impacted survival.

Conclusions These findings confirm that CD11b is an autoimmune susceptibility gene that when mutated can exacerbate the severity of disease on a susceptible genetic background. This work highlights an important role for CD11b in regulating and controlling the progression of inflammation and autoimmune disease.

\section{2 NEW POSSIBILITIES OF HUMAN COMPLEMENT SYSTEM IN DIAGNOSTICS AND ANALYSES OF AUTOIMMUNE DISEASES}

'V Lakhtin*, ${ }^{2} \mathrm{M}$ Naxthn, 'S Andina, 'S Afanasiev, ${ }^{1} \mathrm{O}$ Logunov, 'V Aleshkin. 'G.N. Gabrichevsky research Institute for Epidemiology and Microbiology, Department of Biotechnology, Moscow, Russia; ${ }^{2}$ G.N. Gabrichevsky research Institute for Epidemiology and Microbiology, Department of Biotechology, Moscow, Russia

\subsection{6/lupus-2017-000215.342}

Background and aims The aim was to summarise own data on the human complement system involving cofunctioning 